\title{
Element-based Lattice Reduction aided K-Best detector for large-scale MIMO systems
}

\author{
Ogeen H. Toma and Mohammed El-Hajjar \\ Electronics and Computer Science, University of Southampton \\ Southampton SO17 1BJ, UK \\ meh@ecs.soton.ac.uk
}

\begin{abstract}
Recently, large-scale Multiple-Input MultipleOutput (MIMO) systems have caught great attention for increasing the system throughput as well as improving the system performance. The main challenge in the design of these MIMO systems is the detection techniques used at the receiver. Lattice Reduction (LR) techniques have shown good potential in MIMO decoding due to their good performance and low complexity compared to Maximum Likelihood (ML) detector. The Lenstra, Lanstra, and Lovasz (LLL) LR algorithm has been employed for decoding while combined with linear detectors such as ZF as well as with K-Best detection. However, the LLL-aided detectors have shown limited performance, when increasing the number of antennas at the transmitter and receiver. Therefore, in this paper we propose to use the so-called Element-based Lattice Reduction (ELR) combined with K-Best detector for the sake of attaining a better BER performance and lower complexity than the LLL-aided detection. Explicitly, the ELR-aided detectors are capable of attaining a $2 \mathrm{~dB}$ performance improvement at BER of $10^{-5}$ compared to the LLL-aided detectors when considering a MIMO system with 200 transmit and receive antennas. Furthermore, for the same MIMO configuration, the ELR basis update requires nearly an order of magnitude reduction in the number of arithmetic operations compared to the LLL algorithm.
\end{abstract}

Index Terms-Large-scale MIMO, detection, Lattice Reduction, K-Best detection.

\section{INTRODUCTION}

$\mathbf{T}$ HE continuous growing demand for high data rate wireless services and for higher quality of service (QoS) is pushing wireless communications networks to introduce new technologies. Multiple-Input Multiple-Output (MIMO) techniques have attracted considerable attention due to their potential to increase the data throughput and link range without the need for any additional bandwidth or transmit power [1] [3]. MIMO has been adopted as a key technique in the fourth generation (4G) mobile communications standards. Recently, large-scale MIMOs have been proposed in order to scale up the MIMO gains [4], [5].

In MIMO systems, where multiple interfering symbols are transmitted at the same time, the received symbols are expected to be detected subject to the contamination of random noise or interference. The detection challenge increases with the increase of the number of antennas and hence in largescale MIMO, reducing the detection complexity is one of the main challenges.

978-1-5090-1749-2/16/\$31.00 (C2016 IEEE
Although the Maximum Likelihood (ML) detector offers optimal BER performance, its complexity makes it impractical in terms of hardware implementation. On the other hand, linear detectors such as Zero Forcing (ZF) detection is simple to implement, while it suffers from significant performance degradation compared to the ML. Recently, Lattice Reduction (LR) aided detectors were proposed for MIMO systems, that are capable of achieving a sub-optimal performance close to ML with much less complexity [6], [7]. Several algorithms have been proposed in the literature for performing Lattice Reduction such as the Lenstra, Lanstra, and Lovasz (LLL) algorithm [8], the Korkine-Zolotareff (KZ) algorithm [9] and the Element-based Lattice Reduction (ELR) algorithm [10]. These algorithms vary in complexity and performance. The most widely used is the LLL algorithm due to its efficiency and low complexity, while $\mathrm{KZ}$ requires high computational complexity. On the other hand, the ELR algorithm has been proposed as a reduced complexity LR algorithm, while performing better than the LLL-aided MIMO detectors when the number of antennas in significantly high [10]. In large scale MIMOs, the ELR algorithm requires less number of arithmetic operations for the LR basis update than the LLL algorithm [10].

LLL-aided linear detectors were employed in [7], [11], where the Bit Error Ratio (BER) performance improvement was achieved compared to conventional linear detectors dispensing with LR, while this required a slight increase in the implementation complexity. Furthermore, in order to reduce the performance gap between the LR-aided detectors and the ML detector, [12] proposed an LLL-aided K-Best detector, which was capable of improving the system performance further while increasing the preprocessing complexity.

Recently, the ELR algorithm has been proposed to perform lattice reduction for large-scale MIMO systems in order to attain an improved BER performance while maintaining lower complexity compared to the LLL-aided detector [10]. Therefore, motivated by the results of [12], where the performance of the K-Best detector was improved by performing $L R$ of the channel matrix before the detection, in this paper we propose to improve the large-scale MIMO detection performance by applying the ELR algorithm with the K-Best detector, which we refer to as ELRaided K-Best detector. Our simulation results show that our proposed ELR-aided K-Best detector is capable of achieving an improved BER performance compared to the benchmark techniques utilising the LLL algorithm in the 
LR-aided K-Best detector, while requiring a significantly lower complexity. Explicitly, the ELR-aided detectors are capable of attaining a $2 \mathrm{~dB}$ performance improvement at BER of $10^{-5}$ compared to the LLL-aided detectors when considering a MIMO system with 200 transmit and receive antennas. Furthermore, for the same MIMO configuration, the ELR basis update requires nearly an order of magnitude reduction in the number of arithmetic operations compared to the LLL algorithm.

The rest of the paper is organized as follows. In Section II we present an overview of the MIMO system model used in this paper followed by Section III, where the LR-aided MIMO detection is explained. In Section III, we present our proposed ELR-aided K-Best detector. Then, we present our simulation results in Section IV, where we compare the BER performance as well as the complexity of the proposed detector with the benchmark techniques. Finally, we present our conclusions in Section V.

\section{SYSTEM MODEL}

Consider a MIMO system with $N$ transmit and $M$ receive antennas, where different data symbols are transmitted from the different transmit antennas at the same time. The channel between the transmitter and receiver is assumed to be Rayleigh fading channel, where the different channels between the different transmit and receive antennas are independent and identically distributed (i.i.d.), since it is assumed that the antennas at the transmitter and receiver are sufficiently spaced apart.

Let $\mathbf{x}^{c}$ denote the transmitted complex symbol vector of size $N \times 1$, where $\mathbf{x}^{c}=\left[x_{1}^{c}, x_{2}^{c}, \ldots, x_{N}^{c}\right]^{T}$ such that $x_{i}^{c} \in X^{c}$ is drawn from a complex constellation of P-QAM. Furthermore, the channel can be described by a complex matrix $\mathbf{H}^{c}$ of size $M \times N$, where $\mathbf{H}^{c}$ changes independently from one frame to another. The received signal can be expressed as:

$$
\mathbf{y}^{c}=\mathbf{H}^{c} \mathbf{x}^{c}+\mathbf{n}^{c},
$$

where $\mathbf{y}^{c}=\left[y_{1}^{c}, y_{2}^{c}, \ldots, y_{M}^{c}\right]^{T}$ is the received complex signal vector of size $M \times 1$ and $\mathbf{n}^{c}=\left[n_{1}^{c}, n_{2}^{c}, \ldots, n_{M}^{c}\right]^{T}$ represents the complex Additive White Gaussian Noise (AWGN) vector of size $M \times 1$ with zero mean and variance $\frac{N_{0}}{2}$. The detector at the receiver tries to retrieve the transmitted vector $\mathbf{x}^{c}$ from the received signal $\mathbf{y}^{c}$.

In the previous explanation, we have used the superscript $c$ in the notations representing the transmitted symbol vector, received symbol vector, channel and noise in order to denote that these are complex valued. The complex model of (1) can be represented in an equivalent real model as follows:

$$
\left[\begin{array}{c}
\Re\left[\mathbf{y}^{c}\right] \\
\Im\left[\mathbf{y}^{c}\right]
\end{array}\right]=\left[\begin{array}{c}
\Re\left[\mathbf{H}^{c}\right],-\Im\left[\mathbf{H}^{c}\right] \\
\Im\left[\mathbf{H}^{c}\right], \Re\left[\mathbf{H}^{c}\right]
\end{array}\right]\left[\begin{array}{l}
\Re\left[\mathbf{x}^{c}\right] \\
\Im\left[\mathbf{x}^{c}\right]
\end{array}\right]+\left[\begin{array}{c}
\Re\left[\mathbf{n}^{c}\right] \\
\Im\left[\mathbf{n}^{c}\right]
\end{array}\right],
$$

where $\Re$ and $\Im$ represent the real and imaginary parts of a complex number, respectively. Additionally, (2) can be represented in the following form:

$$
\mathbf{y}=\mathbf{H x}+\mathbf{n} .
$$

Since all the elements of each variable are decomposed into their real and imaginary parts, the equivalent real model of (3) has $\mathbf{y}=\left[y_{1}, y_{2}, \ldots, y_{2 M}\right]^{T}$ and $\mathbf{n}=\left[n_{1}, n_{2}, \ldots, n_{2 M}\right]^{T}$ both of size $2 M \times 1$, while $\mathbf{H}$ has a size of $2 M \times 2 N$, and $\mathbf{x}=$ $\left[x_{1}, x_{2}, \ldots, x_{2 N}\right]^{T}$ of size $2 N \times 1$. In this case, each $x_{i} \in X$ is drawn from the real constellation set of P-QAM as $\{-\sqrt{P}+$ $1,-\sqrt{P}+3, \ldots, \sqrt{P}-1\}$. For example the real model of a given $x^{c}=[1+j, 3-j]^{T}$ of size $2 \times 1$ will be $x=[1,3,1,-1]^{T}$ of size $4 \times 1$. In the following we will use the equivalent real model for the design of the proposed detector.

\section{LR-Aided MIMO Detector Design}

Based on (3) the ML detector requires to search through all possible constellation points of the transmitted symbol vector $\mathbf{x}$ within the lattice $\mathbf{H x}$, which can be expressed as:

$$
\widehat{\mathbf{x}}=\arg \min _{\mathbf{x} \in X^{2 N}}\|\mathbf{y}-\mathbf{H x}\|^{2} .
$$

This process is time consuming and requires complex computations. To reduce this complexity LR-aided detectors were proposed in [6], [7]. LR-aided detectors try to reduce the basis of the lattice $\mathbf{H x}$ and find another basis with better properties for detection [13]. The LR operation transforms the channel matrix $\mathbf{H}$ into its equivalent channel matrix $\widetilde{\mathbf{H}}$, which is more orthogonal and better conditioned than $\mathbf{H}$ [14]. The LR-aided detector uses the new orthogonal channel matrix $\widetilde{\mathbf{H}}$, which may give more reliable estimation for the received signal than that of the detector that uses the original channel matrix $\mathbf{H}$.

The new $\widetilde{\mathbf{H}}$ matrix can be obtained by transforming the MIMO equation as follows:

$$
\begin{aligned}
\mathbf{y} & =\mathbf{H} \mathbf{x}+\mathbf{n} \\
\Rightarrow \mathbf{y} & =\mathbf{H T T}^{-1} \mathbf{x}+\mathbf{n} \\
\Rightarrow \mathbf{y} & =(\mathbf{H T})\left(\mathbf{T}^{-1} \mathbf{x}\right)+\mathbf{n} \\
\Rightarrow \mathbf{y} & =\widetilde{\mathbf{H}} \mathbf{z}+\mathbf{n} .
\end{aligned}
$$

The new channel matrix is generated as $\widetilde{\mathbf{H}}=\mathbf{H T}$, where $\mathbf{T}$ is a uni-modular matrix having a determinant of \pm 1 and integer entries. Multiplying the matrix $\mathbf{T}$ with $\mathbf{H}$ produced the orthogonal matrix $\widetilde{\mathbf{H}}$.

Then, using the model in (5), given by $\mathbf{y}=\widetilde{\mathbf{H}} \mathbf{z}+\mathbf{n}$, the detector requires decoding $\mathbf{z}=\left(\mathbf{T}^{-1} \mathbf{x}\right)$ from the reducedlattice constellation and then recovers the original constellation point by $\widehat{\mathbf{x}}=\mathbf{T z}{ }^{1}$. Both $\mathbf{H x}$ and $\widetilde{\mathbf{H}} \mathbf{z}$ produce the same point in the lattice but $\widetilde{\mathbf{H}}$ is more orthogonal than $\mathbf{H}$.

Various LR algorithms have been proposed in literature in order to produce the $\mathbf{T}$ matrix. In the following we will describe first the LLL algorithm which will form the benchmark to our proposed work. Then, we will describe the ELR algorithm that we utilized in our proposed detector.

\section{A. LLL-Aided ZF detector}

In this section, we present the LLL-aided ZF detector, where the LLL LR algorithm is used to obtain the $\mathbf{T}$ matrix [8], which will result in a new channel matrix $\widetilde{\mathbf{H}}=\mathbf{H T}$. Then, the conventional ZF detection is performed, where the equalisation matrix $\mathbf{W}$ is obtained as the inverse of the new channel matrix

\footnotetext{
${ }^{1}$ Note that $\mathbf{z} \in \mathcal{Z}$ integer set.
} 
$\widetilde{\mathbf{H}}$, i.e. $\mathbf{W}=\widetilde{\mathbf{H}}^{-1}$. This is in contrast to the conventional ZF, where $\mathbf{W}=\mathbf{H}^{-1}$.

Afterwards, $\mathbf{W}$ is multiplied by the received signal using the transformed MIMO model presented in (5). Then, the output of the LLL-aided ZF will be $\widehat{\mathbf{z}}=\mathbf{z}+\widetilde{\mathbf{H}}^{-1} \mathbf{n}$ and since $\mathbf{z}=$ $\left(\mathbf{T}^{-1} \mathbf{x}\right) \in \mathcal{Z}$, the original constellation symbols $\mathbf{x}$ can be recovered by multiplying with $\mathbf{T}$ after shifting, scaling and rounding as follows [7]:

$$
\widehat{\mathbf{x}}=2 \mathbf{T}\left\lceil\left(\widehat{\mathbf{z}}-\mathbf{T}^{-1} \mathbf{1}_{2 N \times 1}\right) / 2\right\rfloor-\mathbf{1}_{2 N \times 1},
$$

where $\mathbf{1}_{2 N \times 1}$ is a $(2 N \times 1)$ vector of all 1 entries.

\section{B. LLL-Aided K-Best detector}

As mentioned in Section I, [12] showed that the performance of the K-Best detector can be improved by performing a LR of the channel matrix as a preprocessing stage prior to the K-Best. The LLL algorithm can be utilised to aid the KBest detector by first producing the $\mathbf{T}$ matrix in order to find the new channel matrix $\widetilde{\mathbf{H}}=\mathbf{H T}$. Then, the K-Best detection process is applied, where the QR decomposition is performed for the new channel matrix as $\widetilde{\mathbf{H}}=\widetilde{\mathbf{Q}} \widetilde{\mathbf{R}}$, with $\widetilde{\mathbf{R}}$ being an upper triangular matrix and $\widetilde{\mathbf{Q}}$ a unitary matrix ${ }^{2}$.

The size of both $\widetilde{\mathbf{Q}}$ and $\widetilde{\mathbf{R}}$ is $2 M \times 2 N$, when the real MIMO model of (3) is considered as described earlier. After the QR decomposition, (5) can be reshaped from $\mathbf{y}=\widetilde{\mathbf{H}} \mathbf{z}+\mathbf{n}$ to

$$
\mathbf{y}=\widetilde{\mathbf{Q}} \widetilde{\mathbf{R}} \mathbf{z}+\mathbf{n} .
$$

Then, the ML detection problem for $\widehat{\mathbf{z}}$ can be expressed as:

$$
\widehat{\mathbf{z}}=\arg \min _{\mathbf{z} \in \mathcal{Z}^{2 N}}\|\check{\mathbf{y}}-\widetilde{\mathbf{R}} \mathbf{z}\|^{2},
$$

where $\check{\mathbf{y}}=\widetilde{\mathbf{Q}}^{H} \widetilde{\mathbf{y}}$ and $\mathbf{z}=\left(\mathbf{T}^{-1} \mathbf{x}\right)$.

Note that

$$
\widetilde{\mathbf{y}}=\left(\mathbf{y}-\mathbf{H} \mathbf{1}_{2 N \times 1}\right) / 2,
$$

which is a shifted and scaled version of $\mathbf{y}$ [15]. From (8), the detector requires performing a Tree Search to detect $\mathbf{z}$ and then recovers the original symbols by multiplying with $\mathbf{T}$ after rescaling and re-shifting $\widehat{\mathbf{z}}$ as

$$
\widehat{\mathbf{x}}=2 \mathbf{T}(\widehat{\mathbf{z}})+\mathbf{1}_{2 N \times 1},
$$

or in terms of (8) it can be expressed as [15]:

$$
\widehat{\mathbf{x}}=2 \mathbf{T} \arg \min _{\mathbf{z} \in Z^{2 N}}\|\check{\mathbf{y}}-\widetilde{\mathbf{R}} \mathbf{z}\|^{2}+\mathbf{1}_{2 N \times 1} .
$$

On the other hand, the LLL-aided K-Best detector has been further improved in [15] by combining the Minimum Mean Square Error (MMSE) regularization with the LLL-aided KBest detector. This is referred to as LLL-aided MMSE K-Best detector, which has an improved performance compared to the LLL-aided K-Best detector. The difference between LLLaided K-Best and LLL-aided MMSE K-Best detectors is that in the MMSE-aided detector the channel matrix $\mathbf{H}$ is replaced

\footnotetext{
${ }^{2} \mathrm{~A}$ unitary matrix has the following property $\widetilde{\mathbf{Q}}^{H}=\widetilde{\mathbf{Q}}^{-1}$, where ${ }^{H}$ represented the Hermitian transpose.
}

TABLE I

ELR ALGORITHM [16]

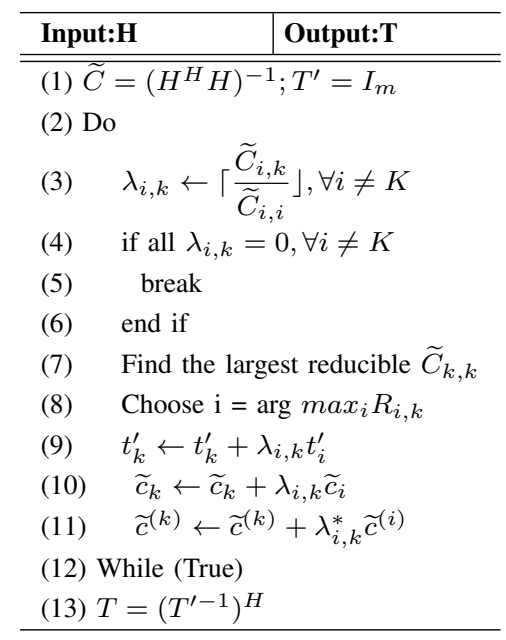

with the extended $\overline{\mathbf{H}}$ matrix and the received signal vector $\mathbf{y}$ is replaced with the extended $\overline{\mathbf{y}}$ as follows:

$$
\overline{\mathbf{H}}=\left[\begin{array}{c}
\mathbf{H} \\
\sqrt{\frac{\sigma^{2}}{p}} \mathbf{I}_{2 N}
\end{array}\right], \overline{\mathbf{y}}=\left[\begin{array}{c}
\mathbf{y} \\
\mathbf{0}_{2 N \times 1}
\end{array}\right],
$$

and then the same K-Best detection process described above is applied.

\section{ELR-Aided ZF detector}

In sections III-A and III-B LLL-aided detectors have been described, which are capable of achieving significant performance improvement compared to decoding whilst dispensing with LR [7], [11]. However, this performance improvement degrades gradually and starts to deviate from the ML performance as the number of antennas increases [10], [12]. This is due to the fact that the LLL algorithm is less efficient in large-scale MIMO system [10], [12]. Recently, Element-based Lattice Reduction (ELR) algorithm [10] has been proposed to perform lattice reduction for large-scale MIMO systems with an improved performance compared to the LLL algorithm, while also requiring lower complexity [10].

The ELR algorithm is shown in Table I, which describes the evaluation process of the matrix T. The ELR algorithm has been applied with ZF detector for large-scale MIMO in [10], [16], where the $\mathbf{T}$ matrix was obtained using the ELR algorithm rather than the LLL algorithm and then ZF detection is employed as described in III-A. A considerable improvement in the BER performance can be noticed in largescale MIMOs, when employing the ELR algorithm compared to the LLL-aided detector, which will be illustrated further in Section IV.

\section{The proposed ELR-Aided K-Best detector}

In this paper, we propose to further improve the performance of ELR-aided detectors by applying the ELR algorithm to aid the K-Best detector, when employed for large-scale 
MIMO systems. Our proposed ELR-aided K-Best detector can achieve an improved BER performance compared to the decoders described in Sections III-A, III-B and III-C, while requiring lower complexity than those employing the LLL algorithm. The ELR-aided K-Best detection process is similar to that described in Section III-B with the difference that it adopts the ELR algorithm described in Table I in order to produce the $\mathbf{T}$ matrix used to obtain the new channel matrix $\widetilde{\mathbf{H}}=\mathbf{H T}$.

As described in Section III-B, consider the MIMO equation given by $\mathbf{y}=\widetilde{\mathbf{H}} \mathbf{z}+\mathbf{n}$, where the K-Best detector can be applied for decoding the received signal. The decoding steps of the ELR-aided K-Best detector can be summarized as follow:

1) Find $\mathbf{T}=E L R(\mathbf{H})$ using the ELR algorithm of Table I;

2) Evaluate $\widetilde{\mathbf{H}}=\mathbf{H T}$;

3) Perform $\mathbf{Q R}$ decomposition for $\widetilde{\mathbf{H}}$ as $[\widetilde{\mathbf{Q}}, \widetilde{\mathbf{R}}]=Q R(\widetilde{H})$;

4) We now have the MIMO model $\mathbf{y}=\widetilde{\mathbf{H}} \mathbf{z}+\mathbf{n}$;

5) Evaluate $\widetilde{\mathbf{y}}=\left(\mathbf{y}-\mathbf{H} \mathbf{1}_{2 N \times 1}\right) / 2$;

6) Evaluate $\check{\mathbf{y}}=\widetilde{\mathbf{Q}}^{H} \widetilde{\mathbf{y}}$;

7) Initialise the variables for performing the K-Best detection as follows $\mathbf{z}=[]$, cost $=0$, len $=1, k=2$;

8) Employ the K-Best detector as described in [15];

9) Evaluate $\widehat{\mathbf{x}}=2 \mathbf{T} \arg \min _{\mathbf{z} \in Z^{2 N}}\|\check{\mathbf{y}}-\widetilde{\mathbf{R}} \mathbf{z}\|^{2}+\mathbf{1}_{2 N \times 1}$.

The proposed ELR-aided K-Best detector can be further enhanced by employing MMSE regularization as described in Section III-C to obtain the ELR-aided MMSE K-Best detector. The difference between the ELR-aided and the ELR-aided MMSE K-Best detection is that the channel matrix $\mathbf{H}$ is replaced with the extended matrix $\overline{\mathbf{H}}$ and also the received signal $\mathbf{y}$ is replaced with the extended $\overline{\mathbf{y}}$ as follows:

$$
\overline{\mathbf{H}}=\left[\begin{array}{c}
\mathbf{H} \\
\sqrt{\frac{\sigma^{2}}{p}} \mathbf{I}_{2 N}
\end{array}\right], \overline{\mathbf{y}}=\left[\begin{array}{c}
\mathbf{y} \\
\mathbf{0}_{2 N \times 1}
\end{array}\right]
$$

The above-proposed ELR-aided K-Best and ELR-aided MMSE K-Best detectors can perform better than the state-ofthe-art detectors including LLL-aided K-Best and LLL-aided MMSE K-Best detectors, while at the same time requiring lower complexity. The reduction in the complexity is mainly due to the fact that the number of the arithmetic operations required by ELR algorithm for basis updates is lower than the LLL algorithm as explained in [10].

\section{Simulation Results}

\section{A. BER Performance}

In this section we present the BER performance of the proposed ELR-aided K-Best and ELR-aided MMSE K-Best detectors and compare these with the benchmark techniques described in Section III, when employed to large-scale MIMO systems. These detectors have been simulated in Matlab for a MIMO system employing $N=200$ transmit and $M=200$ receive antennas, while employing 64-QAM. Additionally, Rayleigh fading channels are considered, where the channels are independent in space and time.

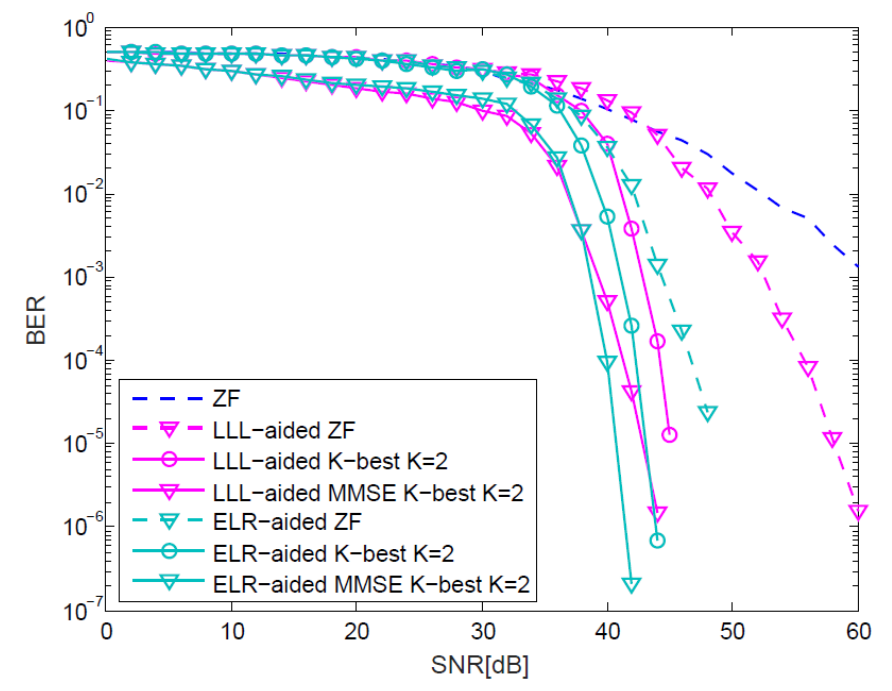

Fig. 1. BER performance of various LR-aided detectors over 200X200 MIMO system and using 64-QAM as a modulation scheme.

Figure 1 shows the BER performance comparison of the various decoding techniques ${ }^{3}$. In Figure 1, we show the BER performance of the ZF detector, which forms as an upper bound on the BER performance of the other detectors. The ZF detector has the lowest complexity, while having the worst BER performance. The LR-aided detectors aim to improve the performance while requiring higher complexity than the ZF detector. The aim of the LR-aided detectors is to attain a suboptimal performance close to that of the ML, while requiring significantly lower complexity.

The simulation results for the LLL-aided detectors show BER performance improvements for the simulated large-scale MIMO compared to the ZF performance. The LLL-aided $\mathrm{ZF}$ detector attains a gain of about $8 \mathrm{dBs}$ at BER of $10^{-3}$ compared to the ZF detector, while the LLL-aided K-Best detector attains a performance gain of around $11 \mathrm{~dB}$ at BER of $10^{-5}$ compared to the LLL-aided ZF detector. Furthermore, the LLL-aided MMMSE K-Best detector attains an additional gain of around $2 \mathrm{~dB}$ at BER of $10^{-5}$ compared to the detector dispensing with MMSE, i.e. the LLL-aided K-Best detector.

On the other hand, the proposed ELR-aided detectors show performance improvement compared to their LLL-aided counterparts. For example, the proposed ELR-aided K-Best detector is capable of attaining a $2 \mathrm{~dB}$ performance gain compared to its LLL-aided counterpart at BER of $10^{-5}$. Additionally, the ELR-aided MMSE K-Best detector outperforms its LLL-aided counterpart by about $3 \mathrm{dBs}$ at BER of $10^{-5}$.

\section{B. Complexity:}

In the following, we illustrate the complexity of the detectors in terms of the number of arithmetic operations including real additions and real multiplications. We compare the complexity of the LLL and the ELR algorithms for performing the LR, where the proposed detector and the benchmark technique are LR-aided detectors and the detection technique

\footnotetext{
${ }^{3}$ Note that in the figure we do not show the performance of the ML detector due to its extremely high complexity for simulation with our configuration.
} 


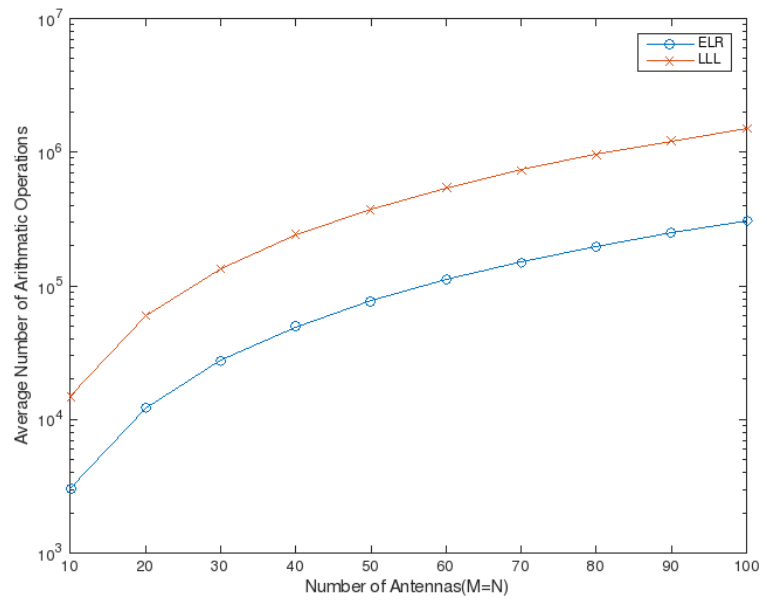

Fig. 2. Average number of arithmetic operations for basis updates of LLL and ELR algorithms versus the number of MIMO transmit and receive antennas [10].

is the same in both detectors except for the LR operation. According to [10], ELR algorithm requires the lowest number of arithmetic operations for basis updates compared to all other LR algorithms. Figure 2 shows the average number of arithmetic operations for the basis update in the LLL and ELR algorithms versus the number of MIMO transmit and receive antennas, where the plot assumes the same number of transmit and receive antennas. As shown in Figure 2, the ELR has a much lower number of arithmetic operations than the LLL algorithm, which is consistent for all number of antennas. The ELR requires nearly an order of magnitude less arithmetic operations that the LLL algorithm, as shown in Figure 2. Therefore, we can conclude that our proposed ELRaided detectors will require significantly lower complexity than their LLL-aided counterparts, while at the same time achieving better performance.

\section{CONClusion}

In this paper, we proposed an ELR-aided K-Best and ELRaided MMSE K-Best detectors that are capable of improving the performance of the LR-aided K-Best detectors for largescale MIMO systems. Our proposed ELR-aided detectors are capable of outperforming their benchmark techniques, while requiring significantly lower complexity. Explicitly, the ELRaided detectors are capable of attaining a $2 \mathrm{~dB}$ performance improvement at BER of $10^{-5}$ compared to the LLL-aided detectors when considering a MIMO system with 200 transmit and receive antennas. Furthermore, for the same MIMO configuration, the ELR basis update requires nearly an order of magnitude reduction in the number of arithmetic operations compared to the LLL algorithm.

\section{REFERENCES}

[1] M. El-Hajjar and L. Hanzo, "Multifunctional MIMO systems: A combined diversity and multiplexing design perspective," IEEE Wireless Communications, vol. 17, pp. 73-79, April 2010.
[2] K. Zheng, L. Zhao, J. Mei, B. Shao, W. Xiang, and L. Hanzo, "Survey of large-scale MIMO systems," IEEE Communications Surveys Tutorials, vol. 17, pp. 1738-1760, third quarter 2015.

[3] L. Hanzo, M. El-Hajjar, and O. Alamri, "Near-capacity wireless transceivers and cooperative communications in the MIMO era: Evolution of standards, waveform design, and future perspectives," Proceedings of the IEEE, vol. 99, pp. 1343-1385, August 2011.

[4] T. Marzetta, "Noncooperative cellular wireless with unlimited numbers of base station antennas," IEEE Transactions on Wireless Communications, vol. 9, pp. 3590-3600, November 2010.

[5] J. Zhang, B. Zhang, S. Chen, X. Mu, M. El-Hajjar, and L. Hanzo, "Pilot contamination elimination for large-scale multiple-antenna aided OFDM systems," IEEE Journal of Selected Topics in Signal Processing, vol. 8, pp. 759-772, Oct 2014.

[6] C. Windpassinger and R. Fischer, "Low-complexity near-maximumlikelihood detection and precoding for MIMO systems using lattice reduction," in IEEE Information Theory Workshop, pp. 345-348, March 2003.

[7] X. Ma and W. Zhang, "Performance analysis for MIMO systems with lattice-reduction aided linear equalization," IEEE Transactions on Communications, vol. 56, pp. 309-318, February 2008.

[8] A. Lenstra, J. Lenstra, H.W., and L. Lovsz, "Factoring polynomials with rational coefficients," Mathematische Annalen, vol. 261, no. 4, pp. 515534, 1982.

[9] E. Agrell, T. Eriksson, A. Vardy, and K. Zeger, "Closest point search in lattices," IEEE Transactions on Information Theory, vol. 48, pp. 22012214, Aug 2002.

[10] Q. Zhou and X. Ma, "Element-based lattice reduction algorithms for large MIMO detection," IEEE Journal on Selected Areas in Communications, vol. 31, pp. 274-286, February 2013.

[11] Y. H. Gan and W. H. Mow, "Complex lattice reduction algorithms for low-complexity MIMO detection," in IEEE Global Telecommunications Conference, vol. 5, pp. 5 pp.-2957, Dec 2005.

[12] X.-F. Qi and K. Holt, "A lattice-reduction-aided soft demapper for highrate coded MIMO-OFDM systems," IEEE Signal Processing Letters, vol. 14, pp. 305-308, May 2007.

[13] C. Ling, "On the proximity factors of lattice reduction-aided decoding," IEEE Transactions on Signal Processing, vol. 59, pp. 2795-2808, June 2011.

[14] P. Silvola, K. Hooli, and M. Juntti, "Suboptimal soft-output MAP detector with lattice reduction," IEEE Signal Processing Letters, vol. 13, pp. 321-324, June 2006.

[15] Q. Zhou and X. Ma, "An improved LR-aided K-best algorithm for MIMO detection," in International Conference on Wireless Communications Signal Processing (WCSP), pp. 1-5, Oct 2012.

[16] Q. Zhou and X. Ma, "Designing low-complexity detectors for generalized SC-FDMA systems," in 45th Annual Conference on Information Sciences and Systems (CISS), pp. 1-6, March 2011. 